\title{
El Ambiente Psicosocial y la Inmunidad Celular en la Paciente Embarazada ${ }^{1}$
}

\author{
Julián A. Herrera M., M.D. ${ }^{2}$, Juan P. Alvarado F., M.D. ${ }^{3}$, \\ y Julia E. Martínez, M.Sc. ${ }^{4}$
}

\section{AGRADECIMIENTOS}

Los autores agradecen al Departamento de Ginecología y Obstetricia, Facultad de Ciencias de la Salud, Universidad del Cauca, Popayán, y a los doctores Oscar Bolaños y Diego Mejía, De-

1. Investigación auspiciada por el Departamento de Ginecología y Obstetricia, Facultad de Ciencias de la Salud, Universidad del Cauca, Popayán, Colombia; por el Decanato de la Facultad de Salud, Universidad del Valle, Cali, Colombia; y por la Sección de Micología Básica, Departamento de Ecología Humana, Facultad de Medicina, Universidad Naciona! de México, México, D.F.

2. Especialista en Medicina Familiar, Profesor Auxiliar, Facultad de Salud, Universidad del Valle, Cali, Colombia, A.A. 4483.

3. Profesor Titular, Departamento de Ginecología y Obstetricia, Facultad de Ciencias de la Salud, Universidad del Cauca, Popayán, Colombia.

4. Inmunóloga Clínica, Universidad Nacional Autónoma de México, México, D.F. cano de la Facultad de Salud y Director Académico Postgrado en Medicina Familiar, respectivamente, Universidad del Valle, Cali, su colaboración y apoyo financiero para la realización de este estudio; al doctor Edgard Cobo, Profesor Titular Emérito, Departamento de Obstetricia y Ginecología, Facultad de Salud, Universidad del Valle, Cali, Colombia, por su asesoría y a la doctora Conchita Toriello, Jefe del Laboratorio de Micología Básica, Universidad Nacional Autónoma de México, México, D.F., por su colaboración para la realización de este estudio. Igualmente los autores agradecen las autorizaciones y asesoría para el uso de las escalas utilizadas, a los doctores Gabriel Smilkstein, Profesor de Medicina Familiar, Universidad de Washington, Seattle, USA, al doctor Thomas Rahe, Profesor de Psiquiatría, Universidad de Nevada, USA y al doctor Max Hamilton, Profesor Titular Emérito de Psiquiatría, Universidad de Leeds, Inglaterra.

McDonald (1) y Erickson (2) asociaron los factores emocionales con las complicaciones obstétricas. Nuckolls et al. (3) demostraron cómo el al to nivel de estrés sin soporte social, se asociaba a complicaciones médicas en $90 \%$ de las embarazadas, en contraste con $33 \%$ de complicaciones 
del embarazo en un grupo de gestantes con buen soporte social. Ramsey (4) describió una asociación estadística entre la estructura y funcionalidad de la familia y recién nacidos de bajo peso, en aquellas embarazadas que no presentaban factores de riesgo para esta condición. Además, se ha demostrado que los factores estresantes alteran la inmunidad celular con disminución de linfocitos $\mathrm{T}$, células Killer y relación de linfocitos $\mathrm{T}$ ayudadores/supresores (5-8). Estos hechos sugieren una posible relación entre el estrés materno y bajo peso al nacer, asi como entre el estrés materno y las infecciones.

En Colombia, las altas tasas de morbimortalidad perinatal se han considerado un problema de salud pública, asociado generalmente a parto prematuro e hipertensión arterial inducida por el embarazo (9), pero no se ha evaluado el papel del ambiente psicosocial con respecto a la inmunidad celular.

En el presente estudio se analizan los cambios en la actividad linfocitaria tanto en pacientes con embarazo normal como asociado con patología, teniendo en cuenta su ambiente psicosocial. Se plantea como hipótesis nula, que una inadecuada relación del estrés y el soporte social, llevaría a una depresión de la inmunidad celular.

\section{PACIENTES Y METODOS}

Se estudiaron 72 pacientes con un margen de error al muestreo de $6 \%$ y un grado de confianza de $95 \%$. De ellas, $38(52.7 \%)$ pacientes presentó patología obstétrica: $32(84.2 \%)$ trabajo de partc prematuro y $6(15.8 \%)$ hipertensión ar terial inducida por el embarazo. Fueron hospitalizadas en la unidad de cuidados especiales del Departamento de Obste- tricia y Ginecología del Hospital Universitario del Valle (HUV) en Cali, Colombia. Las 34 restantes fueron pacientes con embarazos normales que asistían a la consulta externa de los centros de salud La Selva y Siloé de la misma ciudad.

Todas las pacientes tenían el antecedente de candidiasis diagnosticada mediante examen en fresco con $\mathrm{KOH}$, con más de 32 semanas de gestación. Dadas las características del estudio se excluyeron aquellas con alteraciones en la inmunidad celular tales como desnutrición, obesidad, diabetes mellitus, tratamiento con corticosteroides o antecedentes de anafilaxia a antígenos (10-12). Los dos grupos estudiados son comparables desde el punto de vista de edad, paridad y nivel socio-económico.

Se utilizaron formularios precodificados que fueron diligenciados por 3 médicos que desconocían el objetivo del estudio $y$ en los que se evaluaron 14 variables mediante revisión de la historia clínica para datos de identificación y caracterización de las pacientes. Cada entrevistador encuestó un solo grupo de paciente para obviar el conocimiento si era un caso o un control.

En los formularios se valoraron los siguientes puntos:

1. Edad, diagnóstico y antecedentes obstétricos, antecedentes virales durante la gestación.

2. Evaluación de la ansiedad soportada por la paciente antes de presentar la patología motivo de la hospitalización. Los síntomas de ansiedad se valoraron con base en la escala de Hamilton (13, 14) (Cuadro 1), teniendo en cuenta únicamente los síntomas previos al cuadro patológico, para evitar los po- 


\section{CUADRO 1 \\ Escala de Ansiedad de Hamilton}

Clave: $0=$ Ausente $1=$ Intensidad leve $2=\ln$ tensidad media $3=\operatorname{lntensidad~fuerte~} 4=\ln$ tensidad máxima (incapacitante)

Item

1. Humor ansioso

2. Tensión

3. Miedos

4. Insomnio

5. Funciones intelectuales (Gnósicas)

6. Humor depresivo

7. Síntomas somáticos

8. Sintomas somáticos generales (sensoriales)

9. Sintomas cardiovasculares

10. Respiratorios

11. Sintomas gastrointestimales

12. Sintomas genito-urinarios

13. Síntomas del sistema nervioso autónomo

14. Comportamiento durante la entrevista
Inquietud, espera de lo peor, aprensión

$\begin{array}{lllll}0 & 1 & 2 & 3 & 4\end{array}$

(anticipación temerosa), irritabilidad

Sensación de tensión, fatigabilidad, sobresaltos, llanto fácil, temblor, sensación de no poder quedarse en un lugar, incapacidad de relajarse.

A la oscuridad, a la gente desconocida, a quedarse $\quad \begin{array}{lllll}0 & 1 & 2 & 3 & 4\end{array}$ solo, a los animales, al tráfico, a la multitud.

Dificultad en conciliar el sueño. Sueño inter rumpido, sueño no satisfactorio con cansancio al despertar, sueños penosos, pesadillas, terrores nocturnos.

Dificultad de concentración, mala memoria

$\begin{array}{llll}0 & 1 & 2 & \dot{3} \\ \cdot & 4\end{array}$

Falta de interés, no disfruta ya con los pasatiempos, depresión, insomnio de la madrugada, variaciones de humor durante el día.

Dolores y cansancio muscular, contracciones, rigidez, sacudidas mioclónicas, chirrido de dientes, voz poco firme, tono muscular aumentado.

Zumbidos de oídos, visión borrosa, sofocos o escalofríos, sensación de debilidad, sensación de hormigueo.

Taquicardia, palpitaciones, dolores en el pecho latidos vasculares, sensación de desmayo, extrasístoles

Peso u opresión torácica, sensación de ahogo, suspiros, disnea

Dificultad para tragar, meteorismo, dolor abdominal, sensación de ardor, plenitud abdominal, náuseas, vómitos, borborigmo, sensación de estómago vacío, pérdida de peso, estreñimiento.

Micciones frecuentes, urgencia de micción, amenorrea, menorragia, desarrollo de frigidez, eyaculación precoz, pérdida de la líbido, impotencia.

Boca seca, accesos de rubor, palidez, transpiración excesiva, vértigo, cefalea de tensión, piloerección

Agitado, inquieto o dando vueltas. manos temblorosas, ceño fruncido, facies tensa, suspiros o respiración agitada, palidez, tragar saliva; eructus, rápidos movimientos de los tendones, midriasis, exoftalmos. 
sibles efectos psicológicos de la hospitalización o efectos farmacológicos indeseables.

3. Valoración de la función de la familia, con base en el Apgar Familiar de Smilkstein $(15,16)$ (Cuadro 2).

4. El soporte social recibido en el embarazo se valoró mediante preguntas directas que han sido validadas en otros estudios $(3,17,18)$ con las cuales se evaluó la satisfacción por el afecto $y$ apoyo recibidos por su familia en el embarazo, aceptación personal y familiar al embarazo, integración social o aislamiento en el embarazo.

5. Valoración de la actividad linfocitaria mediante la prueba de hipersensibilidad tardía para la candidina (Dermatophy ton-O) correlacionado al recuento absoluto de linfocitos en extendido periférico (19). El antígeno debidamente estandarizado se obtuvo del Laboratorio de Micología Básica de la

\section{CUADRO 2}

\section{Cuestionario de Apgar Familiar*}

\begin{tabular}{|c|c|c|c|c|}
\hline Componente & Pregunta & $\begin{array}{l}\text { Casi } \\
\text { siempre }\end{array}$ & $\begin{array}{l}\text { Puntaje } \\
\text { Algunas } \\
\text { veces }\end{array}$ & $\begin{array}{l}\text { Casi } \\
\text { nunca }\end{array}$ \\
\hline
\end{tabular}

\section{Adaptación}

Cómo se comparten los recursos, satisfacción por la ayuda recibida por la familia.

Cómo se comparten las decisiones o la satisfacción del miembro de la familia en la comunicación familiar y solución de los problemas.

Apoyo de los miembros de la familia ante los cambios en el estilo de vida.

\section{Afecto \\ Cómo se comparten las experiencias emocionales, o la satisfacción con la intimidad y la interacción emocional:}

Cómo se comparte el tiempo, espacio y dinero.
¿Estoy satisfecho con la ayuda que recibo de mi familia cuando algo me preocupa?

¿Estoy satisfecho con la forma en que mi familia discute asuntos de interés común, y comparte la solución del problema conmigo?

¿Mi familia acepta mis deseos para promover nuevas actividades o hacer cambios en mi estilo de vida?

¿Estoy satisfecho con la forma en que mi familia expresa afecto y responde a mis sentimientos de ira, amor y tristeza?

¿Estoy satisfecho con la cantidad de tiempo que mi familia y yo compartimos juntos?

\section{2}

1

0

1

0

2

1

0

2

0

2

10

Puntaje: 7 - 10: Alta funcionalidad familiar

4- 6 : Sugiere disfunción familiar moderada

$0-3$ : Sugiere disfunción familiar severa

* Con permiso de Smilkstein, C.: Family Practice, 1978, 6: 1231-1239. 
Universidad Nacional Autónoma de México (lote MI-55). La dosis de antígeno utilizada fue de $0.1 \mathrm{cc}$ por vía intradérmica y se interpretó a las 48 horas, considerando la prueba como positiva con un eritema e induración igual o mayor a $5 \mathrm{~mm}$ de diámetro.

La información se obtuvo de mayo a septiembre de 1986; en este lapso se hizo una medida de consistencia por muestreo sistemático de 8 formularios precodificados con un margen de error de $6 \%$ y un grado de confianza de $95 \%$, los cuales fueron diligenciados en forma ciega para confrontar los puntajes de las variables, encontrando consistencia en la información recogida.

\section{RESULTADOS}

Del total de las pacientes 31 (43\%) eran primigestantes y $41(57 \%)$ multíparas, con una edad promedio de 23 años, uscilando $76.4 \%$ de las pacientes entre 18 a 35 años. En la evaluación de la ansiedad con base en la escala de Hamilton cuyo rango es de 0 a 56 puntos, con mayor puntaje cuanto mayor sea la ansiedad, se encontró que la ansiedad severa (Hamil- ton $>25$ puntos) fue más frecuente en las pacientes patológicas con una diferencia estadísticamente significativa $(p<0.01)$.

\section{Nivel de estrés y soporte social}

Se consideró nivel alto de estrés cuando la paciente tenía más de 5 variables estresantes, encontrándose en 20 $(52.6 \%)$ de las pacientes patológicas y en $6(20.5 \%)$ de las embarazadas normales $(p<0.001)$, presentando un riesgo relativo para desarrollar patología obstétrica de 4.2 (ic: 3.6-4.8). La asociación de alto nivel de estrés con ausencia de soporte social al embarazo se encontró en $8(21.0 \%)$ de las pacientes patológicas y en $1(2.9 \%)$ de las pacientes del grupo control $(p<0.05)$, aumentando el riesgo relativo para desarrollar patología obstétrica a 9.0 (ic: 2.5-41.7) cuando, además del alto nivel de estrés, se carece de soporte social adecuado para el embarazo.

\section{Antecedentes virales}

Analizando los antecedentes virales durante la gestación, se encontró mayor

\section{CUADRO 3 \\ Variables Estresantes Estudiadas}

1. Ansiedad severa. Puntaje de Hamilton $>25$.

2. Eventos de cambio acumulativos, con puntaje $>300$ según la escala de reajuste social de Holmes y Rahe.

3. Embarazo no deseado, con algún grado de ansiedad valorado con un puntaje de Hamilton $>2$.

4. Madre soltera, con algún grado de ansiedad valorado con un puntaje de Hamilton $>2$.

5. Temor anticipatorio al parto o a malformaciones fetales, con algún grado de ansiedad valorada. con un puntaje de Hamilton $>2$.

6. Condiciones socioeconómicas inadecuadas:

Vivienda sin área adecuada para la familia nuclear.

Alimentación no adecuada para las necesidades fisiológicas de la gestante.

Ingresos económicos inadecuados para la familia nuclear con base en el salario mínimo vigente y el costo de la canasta familiar. 
incidencia para herpes tipo I y condilomas acuminados en el grupo patológico con respecto al control, siendo de $23.6 \%$ y de $14.7 \%$ respectivamente, sin diferencias estadísticamente significativas entre los grupos.

\section{Prueba de candidina}

Analizando la respuesta de hipersensibilidad tardía para la candidina, se encontró que $13(38.2 \%)$ de las embarazadas normales eran negativas a la prueba de candidina y $19(50 \%)$ de las embarazadas patológicas tenían la prueba de candidina negativa. La asociación de nivel al to de estrés sin soporte social al embarazo presentó una prueba de candidina negativa en $100 \%$ de las pacientes, comparado con una prueba de candidina negativa en $19(40.4 \%)$ de las pacientes con nivel bajo de estrés y buen soporte social al embarazo.

\section{Recuento de linfocitos}

Considerando la linfopenia en la embarazada como un recuento inferior a 1.500 linfocitos en extendido periférico, se encontró que $31.5 \%$ de embarazadas con patología presentaban linfopenia, y se halló únicamente en $5.8 \%$ de las embarazadas normales ( $p<0.01$ ). Ell92.9:\% de pacientes con linfopenia absoluta tenían una prueba de candidina negativa.

\section{DISCUSION}

En la hipertensión arterial inducida por el embarazo se ha demostrado una hiporreactividad inmunológica frente a un exceso de antígenos de histocompatibilidad paternos, evidenciado por la ausencia de anticuerpos HLA, déficit de $\operatorname{lgG}$ e IgM y menor efecto bloqueante en el suero de la madre (20), que podría explicar la mayor frecuencia en primigestantes, diabéticas, fetos macrosómicos o con triploidias, y la menor frecuencia de toxemia después del cambio de cónyuge o del aborto.

En humanos el estrés produce disminución de actividad de linfocitos $\mathrm{T}$ y células Killer (5). Por este mecanismo se ha explicado cómo el estrés en animales de experimentación aumenta la susceptibilidad a infecciones virales (21), disminuye la respuesta de hipersensibilidad tardía mediada por linfocitos $\mathrm{T}$ (22) y disminuye el título de anticuerpos a estímulos específicos como la fitohemaglutinina A (7). Como es conocido, la respuesta inmune está bajo control genético y del sistema nervioso central, específicamente el hipotálamo (23-25), lo cual podría explicar la tendencia familiar en la toxemia, y la disminución de actividad de linfocitos $T$ y células Killer que se ha visto en la paciente toxémica con relación a la embarazada normal (20).

En las pacientes estudiadas se encontró una asociación estadística entre los factores emocionales, la angustia severa y el alto nivel de estrés, aumentándose en más de 9 veces el riesgo relativo para desarrollar patología obstétrica cuando concomitantemente se carece de un adecuado soporte social al embarazo $(28,29)$, tal como se ha descrito en otros estudios $(1-4,18,26-30)$.

La respuesta de hipersensibilidad tardía fue negativa en mayor proporción en las pacientes con alto nivel de estrés con respecto a las pacientes con un ambiente psicosocial adecuado. Estos resultados concuerdan con los estudios experimentales en los cuales la hipersensibilidad tardía se disminuía significativamente en animales estresados con relación a los no estresados (22). 
En las pacientes con patología obstétrica, se encontró mayor proporción de linfopenia $(p<0.01)$, que se asoció en más de $90 \%$ de los casos a una prueba de candidina negativa, indicando compromiso de la actividad linfocitaria en este grupo de pacientes, si tenemos en cuenta que la hipersensibilidad tardía cuantificada es mediada por linfoquinas (31) y la mayor proporción de linfocitos circulantes en esta edad gestacional son del tipo $\mathrm{T}$ (32), responsables de la vía eferente de la intradermorreacción.

Los estímulos psicosociales, las influencias ambientales tempranas y los factores genéticos al interactuar de manera dinámica dentro de una visión sistémica (33), facilitarían mediante mecanismos neuroendocrinos la depresión de la actividad linfocitaria en la paciente embarazada (5). Si tenemos en cuenta que el sistema de aclaramiento de complejos inmunes es propio de la especie humana al igual que la toxemia (20), y que la conducta por efecto hipotalámico puede condicionar inmunosupresión como se ha demostrado en animales de experimentación (25), se podría pensar que la depresión en la actividad linfocitaria demostrada en el $100 \%$ de las pacientes que estaban sometidas a alto nivel de estrés sin soporte social a su embarazo, estaría relacionado con el riesgo relativo que se ha descrito para desarrollar patología obstétrica en las pacientes con esta condición $(3,30,34-36)$.

Dentro del modelo teórico de enfermedad producida por mediación psicosocial impulsado por el doctor Levi del Laboratorio de Estrés de la Universidad Karolinska de Estocolmo, el ambiente psicosocial adverso, de manera lineal (37), produciría alteración en la actividad linfocitaria de la paciente embarazada, que en interacción dinámica (33) facilitaría la génesis de precursores de enfermedad (37-42), lo cual podría aplicarse preventivamente dentro de un modelo biopsicosocial de atención a la gestante $(43,44)$, dando mayores elementos para considerar el bajo nivel socioeconómico y la adolescencia (45-47) como factores de riesgo en el embarazo cuando estos factores generadores de estrés social y familiar no tienen el soporte social adecuado en su nicho ambiental.

\section{RESUMEN}

Se estudiaron 72 pacientes con más de 32 semanas de embarazo, 38 (52.7\%) con trabajo de parto prematuro o hipertensión arterial inducida por el embarazo, hospitalizadas en la unidad de cuidados especiales del Hospital Universitario del Valle, Cali, Colombia, làs 34 (47.3\%) restantes con embarazos normales en los centros de salud de Siloé y La Selva de la misma ciudad. Se valoró el nivel de estrés $y$ el soporte social recibido por la paciente en su embarazo, al igual que la actividad linfocitaria cuantificada mediante el recuento absoluto de linfocitos en extendido periférico y la prueba de hipersensibilidad tardía para la candidina, teniendo todas las pacientes el antecedente de candidiasis demostrada por examen en fresco con $\mathrm{KOH}$.

Se encontró mayor nivel de estrés en las pacientes con patología obstétrica $(p<0.001)$, siendo el riesgo relativo para desarrollar patología de 4.2 (intervalo de confianza (ic) 3.6 - 4.8) cuando la paciente presentaba alto nivel de estrés, que se aumentó a 9 (ic 2.5 - 41.7) cuando la paciente, además del al to nivel de estrés, no tenía el soporte social adecuado para su embarazo. La linfopenia absoluta fue más frecuente en las pacientes patológicas 
$(p<0.01)$, asociado en $92.9 \%$ de las pacientes a una prueba de candidina negati$\mathrm{va}$, teniendo $100 \%$ de las pacientes con alto nivel de estrés sin soporte social a su embarazo, depresión en la actividad linfocitaria demostrada por una prueba de candidina negativa, asociada a linfopenia absoluta en el extendido periférico $(<1.500$ linfocitos).

Los estímulos psicosociales, las influencias ambientales tempranas y los factores genéticos, al interactuar de manera dinámica, facilitan en las pacientes con

\section{SUMMARY}

Seventy two patients with thirty two weeks of pregnancy were studied, thirty eight $(52.7 \%)$ with work of premature parturition or arterial hipertension induced by pregnancy, placed in the unit of special cares of the University Hospital of Valle, Cali, Colombia, the remnant thirty four $(47.3 \%)$ with normal pregnancies in the health centers of Siloé and La Selva of same city. The stress level and the social support received by the patient in her pregnancy were evaluated, as well as the linfocitary activity quantified by the absolute enumeration of linfocites in peripheric extent and the test of late hipersensibility to the candidine, having all the patients the antecedent of demostrated candidiasis shown by examination in fresh with $\mathrm{KOH}$.

A higher level of stress was found in patients with obstetric pathology ( $p<$ 0.001 ), being the relative risk to develop pathology of 4.2 (interval of confidence (ic) 3.6 - 4.8) when the patient showed high stress level, which increased to 9 (ic 2.5 - 41.7) when the patient, besides the high stress level, hadn't the adequate al to nivel de estrés sin soporte social a su embarazo, depresión en la actividad linfocitaria, lo cual podría relacionarse con el riesgo relativo que se ha descrito en este grupo de pacientes para desarrollar patología obstétrica.

De esta manera se dan mayores elementos para considerar el bajo nivel socioeconómico y la adolescencia como factores de riesgo en el embarazo, cuando estos factores generadores de estrés no tienen el soporte social adecuado en sus ambientes familiar o comunitario.

social support for her pregnancy. The absolute linfopenia was more frequent in pathological patients $(p<0.01)$, associated in $92.9 \%$ of patients under negative candidina, having $100 \%$ of patients with high stress level without social support for her pregnancy, depression in the linfocitary activity demonstrated by a test of negative candidine, associated to an absolute linfopenia in the peripheric extent ( 1.500 linfocites).

Psychosocial stimulations, the early environmental influences and genetic factors, on interacting in a dynamic manner, facilitate in patients with a high stress level without social support to their pregnancy, depression in the linfocitary activity, which could be associated with the relative risk that has been described in this group of patients to develop obstetrical pathology.

In this way mayor elements are given to consider the low economical level and adolescence as factors of risk in pregnancy when these factors generators of stress haven't the adequate social support in their familiar or communitary environment. 


\section{BIBLIOGRAFIA}

1. MCDONALD, R. L.: The role of emotional factors in obstetric complication: A review. Psychosom Med, 1968, 30: 222-237.

2. ERICKSON, M. T.: Relationship between psychological attitudes during pregnancy and complication of pregnancy, labor and delivery. Proc Annu Meet Amm. Psychopathol Assoc, 1965, 1: 213-214.

3. NUCKOLLS, K. B., CASSEL, J. \& KAPLAN, B. H.: Psychosocial assets life crisis and the prognosis of pregnancy. Am.J.Epidemiol, 1972, 95: 431-441.

4. RAMSEY, C. H.: The relationship between family functioning, life events, family structure, and the outcome of pregnancy. J. Fam. Pract, 1986, 22 : 521.525.

5. KIELCOT, J., GARDNER, W., SPEICHER, C. et al.: Psychosocial modifiers of immunocompetence in medical students. Psychom Med, 1984, 46: 7-14.

6. BARTROPH, R. W.: Depresead Iynfocite function after bereavement. Lancet, 1979, 1: 1834-1836.

7. SCHLEIFER, S. J., STEVEN, E. K., CAMERINO, M. et al.: Supression of lynfocite stimulation following bereavement. JAMA, 1983, 250: 374-377.

8. YOUNG, M., GEHA, R.: Human regulatory T cell subjets. Ann. Rev.Med, 1986, 37: 165-172.

9. MORENO, C. M.: El problema perinatal en Colombia, pp. 43-50. Memorias del Congreso de Perinatología, Bogotá, 1984.

10. OMS, Informe de un grupo científico: Respuestas inmunológicas de base celular, 1973, 69: 40-45.

11. OMS, Informe de un grupo científico: Inmunidad celular y respuesta a infecciones, 1973, 69: 26-40.
12. OMS, Informe de un grupo científico: Mecanismos de inmunodeficiencia, 1973, 69: 74-85.

13. HAMILTON, M.: The assesment of anxiety tales by rating. Br. Med.J.1959, 32: $50-54$.

14. HAMILTON, M. A.: A rahy scale for depression. J. Neurol Neurosurg Psychiatry, 1960, 23: 561-565.

15. SMILKSTEIN, G.: The family apgar: approach for a family function test and its use by physicians. J. Fam. Pract, 1978, 6: 1231-1239.

16. GOOD, M. J. D. \& SMILKSTEIN, G.: The apgar: A study of constructive validity. J. Fam.Pract, 1979, 8: 577.

17. RAHE, R., MEYER, M.: Social stress and ilness onset. J.Psychiatr. Res., 1964, 8: 35-44.

18. NORBECK, J. S.: Life stress, social support and emotional disequilibrium in complication of pregnancy. A prospective multivariaty study. J. Health Soc Behav, 1983, 24: 30-46.

19. KATZ, P.: Clinical immunology I. Clin. Médicas de NA, 1985, 69: 3.

20. NED , J.: Fenómenos inmunológicos de la toxemia preeclámptica. Clin. Obst. Ginecol, 1979, 3: 443-460.

21. JENSEN, M.\& RASMUSSEN, F.: Stress and susceptibility to viral infection. J. Immunol, 1963, 90: 21-23.

22. PITKIN, D.: Effects of psychological stress on the delayed hipersensivity reaction. Proc.Soc. Exp. Biol.Med, 1965 , 120: 350-351.

23. PROGERS, M., DEVENDRA, M.: The influence of the psyche and the brain on immunity and disease susceptibility: A critical review. Psychosom Med, 1979, 41: 147-164. 
24. ADER, R.: Psychosomatic and psychoimmunology research. Psychosom Med, 1980, 42: 307-322.

25. ADER, R.: Behaviorally conditioned immunosupression. Psychosom Med. 1975, 37: 333-340.

26. GUNTER' L. M.: Psychopatology and the stress in the life experiences of mothers of premature infants. Am J. Obstet. Gynecol, 1963, 86: 333-340.

27. MCDONALD, R. \& CRISTAKOS, A.: The relationship of emotional readjustment during pregnancy to obstetric complication. Am J Obstet Gynecol, 1963, 86: 341-348.

28. GORSUCH, R. \& MARTHAKEY, N.: Abnormalities of pregnancy as a function of anxiety and life stress. Psychosom Med, 1974, 36: 352-362.

29. LANKARAN, V. H. \& VANDENBERG, B. J.: The relationship of maternal altitude to pregnancy outcomes and obstetric complication. Am.J.Obstet.Gynecol, 1980, 136: 374-376.

30. HERRERA, J. A. \& GONZALEZ, M.: El ambiente psicosocial y las complicaciones obstétricas. Colombia Med, 1987, 18: (en prensa).

31. ORTEGA, A.: Inmunopatología, mecanismos generales.

32. GALLS, N.: Aspectos inmunológicos del embarazo normal. Clin.Obstet.NA, 1984, 629-641.

33. BERTALANFY, L.: Perspectivas en la teoría general de sistemas. pp. 10-35, Ed. Alianza Universidad, 1982.

34. EDWARDS, R. G. \& COOMBS, RRA.: Immunological interactions Between mother and fetus. Clinical aspects of immunology. 3 ed., Backewll Oxford, 1974.
35. RIM, J. et al.: Natural killer like activity mediated by Iynfocites, Cell Immunol, 1984, 87: 504-516.

36. FOLCH, H. \& WAKSMAN, H.: The splenic suppresor cell. J.Immunol, 1974, 113: 127-139.

37. THORPE, W. H.: El reduccionismo en la biología. Ed. Ayala, F. J. pp. 152-165, 1983.

38. THURLOW, J.: General susceptibility to ilness. Can. Med. Assoc.J. 1967, 97: 1397-1404.

39. PETRICH, J. \& HOLMES, T. H.: Cambios de vida y comienzo de enfermedad. Clin.Med.NA, 1977, 61: 825-838.

40. HOMES, T. H. \& RAHE, R. H.: The social readjustment rating scales. J.Psychosom Res, 1967, 11: 213-218.

41. LYNCH, J. \& CONVEY, W.: Loneliness, disease and death: alternative approaches Psychosom Med, 1979, 20: 702-708.

42. RAHE, C. \& RAMSON, J. A.: Life change and ilness studies past histories and future directions. J.Human Stress, 1978, 3-15.

43. SMILKSTEIN, G., HELSPPER, A., AINSWORTH, C. et al.: Prediction of pregnancy complications: An application of the biopsichosocial model. Soc. Sci. Med, 1984, 18: 315-321.

44. SMILKSTEIN, G.: Psychosocial risk assesment in clinical medicine. J. Fam. Pract, 1986, 22: 495-496.

45. ALONSO, A. R. de: Madres solteras adolescentes, pp. 10-86, Bogotá, Plaza \& Janés, 1986.

46. CAPRARO. V.: Adolescencia y embarazo. Clin. Obstet.Norteamérica, 1978, 4: 1999-1286.

47. AINSWORTH, M., SALTER, D.: Variables influencing the development of attachment. Oxford University Press, 1975, London, pp. 43-50. 